\title{
Computer modeling influence lines of the bridge crane rods load truss in Mathcad system
}

\author{
Evgenij Kudryavcev*, Aleksandr Gavrilenko, and Mostafa Djafari \\ Moscow State University of Civil Engineering, 129337, Moscow, Russia
}

\begin{abstract}
Article describes modeling processes influencing the lines in rods load truss of the bridge crane in Mathcad system: for the rods of the top belt of a truss; for the rods of the bottom belt of a truss; for diagonal and vertical rods of a truss at various section of the load truss; algorithm and a program complex for automation of modeling and graphical representation influence lines and maximum their values. The proposed modeling procedures of the influence lines in any section of a load truss make it possible to reduce time and cost for these calculations' performance at least in ten times and to carry out the research on load truss parameters, rods parameters and materials change effectively.
\end{abstract}

\section{Introduction}

Computer modeling intended for decision of the diversified problems are receiving more and more propagation [1-14]. Wide use of computer modeling especially at the earliest stages of products, processes and systems creation allow to reduce in many times huge losses at the next stages of cycle life. The article describes process of influence lines' computer modeling in various forms: analytical; program and graphic in any section of the load truss determining their maximum and minimum values of internal efforts.

\section{Theoretical basis and computer modeling in system Mathcad}

Scheme of a load truss of the bridge crane (Figure 1) is shown below.

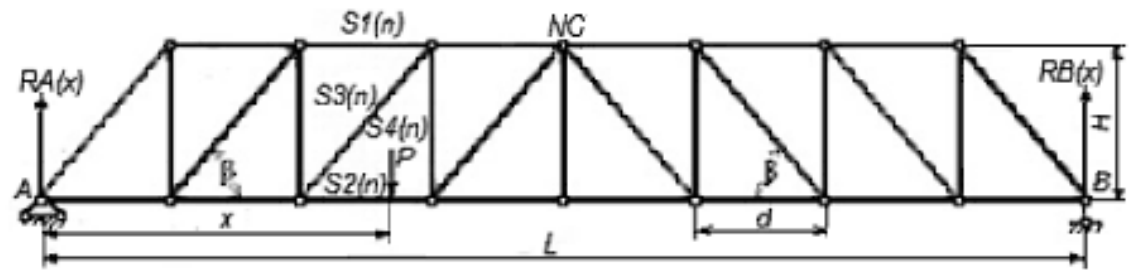

Fig. 1. Scheme of a bridge crane load truss

Input data:

- length and height of load truss, $m$

$L:=14.4 \quad H:=2.4$

* Corresponding author: sdm@mgsu.ru 
- number of sections and influence line grid

$$
H:=8 \quad k:=5
$$

- unit force, $\mathrm{kN}$

Process of modeling influence lines of the bridge crane load truss rods includes some stages:

1. Length section $-d$, influence lines for vertical reactions $R B(x)$ and $R A(x)$ at supports $\mathrm{A}$ and $\mathrm{B}$ : middle point of truss $-\mathrm{NC}$ and a diagonal rod inclination corner sin:

$$
\begin{aligned}
& i:=0 . . L \cdot k \quad Y 0_{i}:=0 \quad d:=\frac{L}{N} \quad x_{i}:=\frac{i}{k} \\
& R B(x):=P \cdot \frac{x}{L} \quad R A(x):=P \cdot \frac{L-x}{L} \quad N C:=\frac{N}{2} \quad \sin :=\frac{H}{\sqrt{H^{2}+d^{2}}}
\end{aligned}
$$

where: $P$ is a unit load;

$x$ is current displacement of unit load on bottom belt of load frame.

2. Influence lines F1(n) for the rods of the top belt of a truss S1(n) in any section n:

- in program form (program 1)

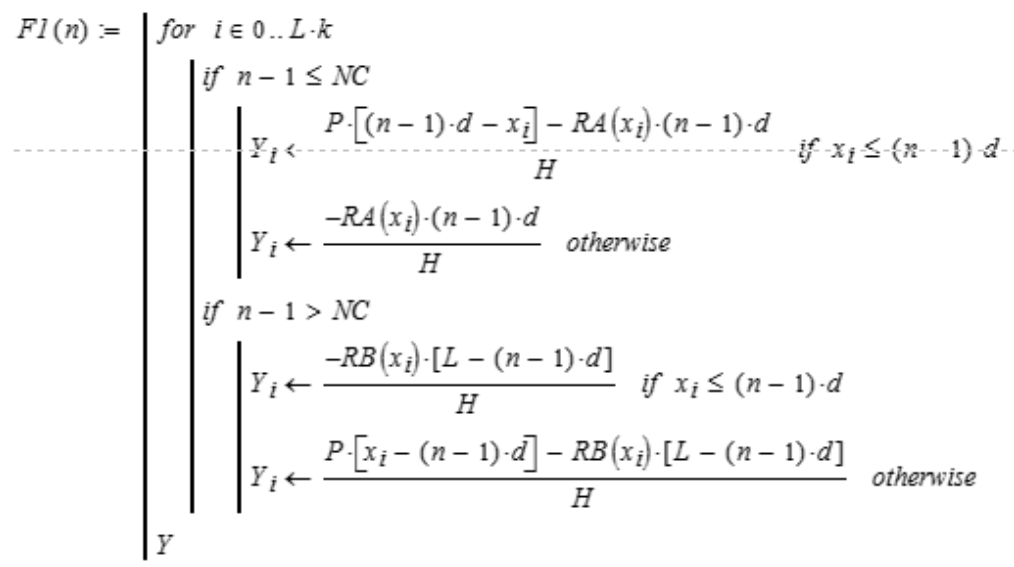

Prog. 1. Program of the influence lines modeling $-F 1(n)$ in the section $\mathrm{n}$ from the position of unit load $x$.

- in a graphical form, for example, in the section (Figure 2)

$$
n:=2
$$

$$
\begin{aligned}
& R A 1:=\frac{-(n-1) \cdot d}{H} \cdot R A(x) \quad R B 1:=\frac{-[L-(n-1) \cdot d]}{H} \cdot R B(x) \\
& \max (F 1(n))=0 \quad \min (F 1(n))=-0.656
\end{aligned}
$$




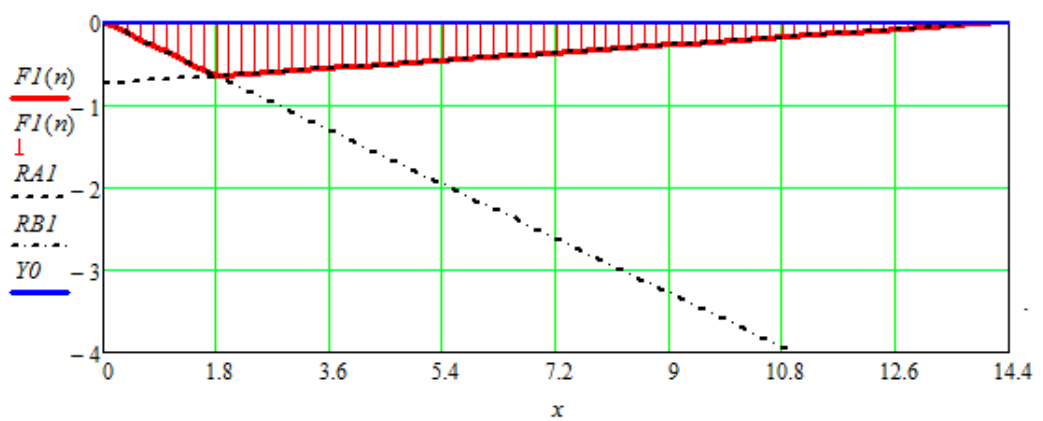

Fig. 2. Influence line $F 1(n)$ for the section $n=2$

3. Influence lines F2(n) for the rods of the top belt of a truss S2(n) in any section n:

- in program form (program 1)

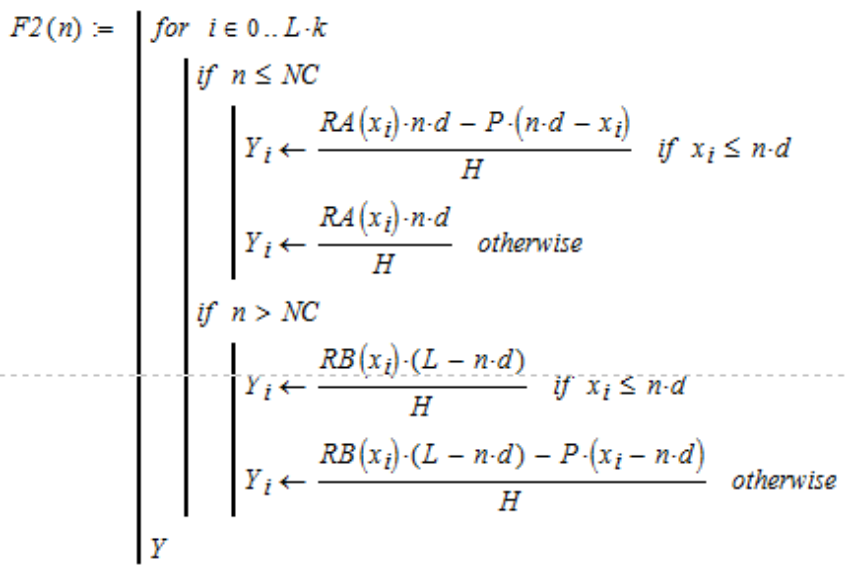

Prog. 2. Program of the influence lines modeling $-F 2(n)$ in the section $\mathrm{n}$ from the position of unit load $x$.

- in a graphical form, for example, in the section (Figure 3)

$$
n:=1
$$

$$
\begin{aligned}
& R A 1:=\frac{n \cdot d}{H} \cdot R A(x) \quad R B 1:=\frac{L-n \cdot d}{H} \cdot R B(x) \\
& \max (F 2(n))=0.656 \quad \min (F 2(n))=0
\end{aligned}
$$

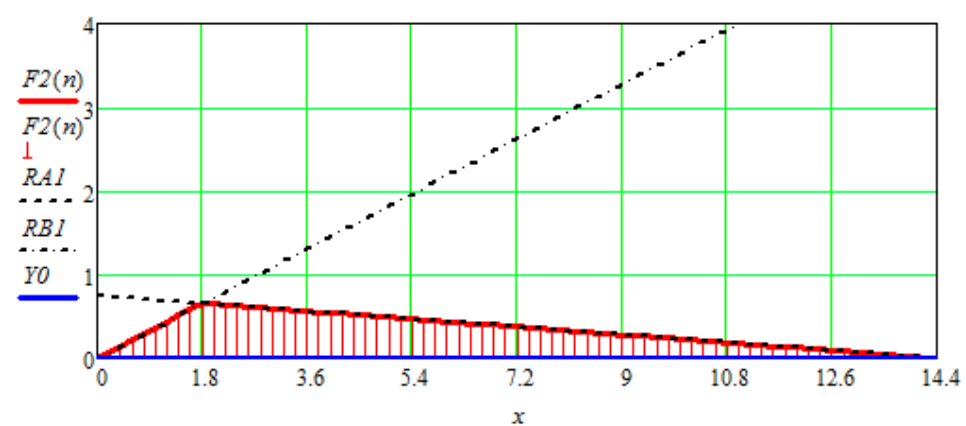

Fig. 3. Influence line F2(n) for the section $n=1$ 
4. Influence lines F3(n) for the diagonal rods of a truss S3(n) in any section n:

- in program form (program 3)

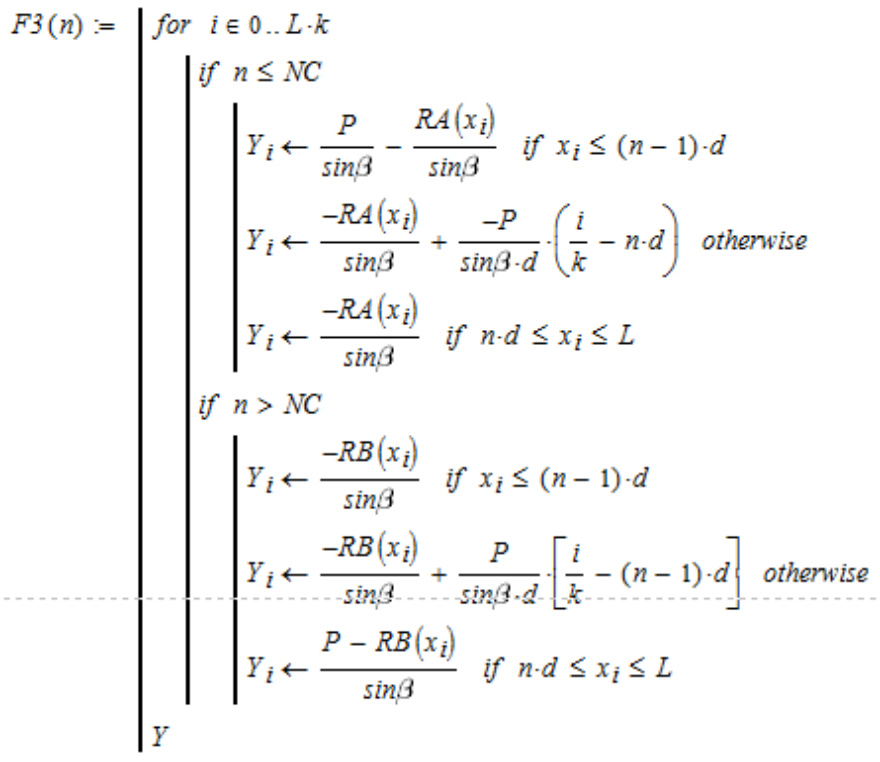

Prog.3. Program of the influence lines modeling $-F 2(n)$ in the section $n$ from the position of unit load $-x$.

- in a graphical form, for example, in the section (Figure 4)

$$
n:=3
$$

$$
\begin{aligned}
& R A 1:=\frac{R A(x)}{\sin \beta} \quad R B 1:=\frac{R B(x)}{\sin \beta} \quad R:=\operatorname{round}\left(\frac{P}{\sin \beta}, 2\right)=1.25 \\
& R A 2:=R A 1-R \quad R B 2:=R B 1-R \quad \max (F 3(n))=0.312 \quad \min (F 3(n))=-0.781
\end{aligned}
$$

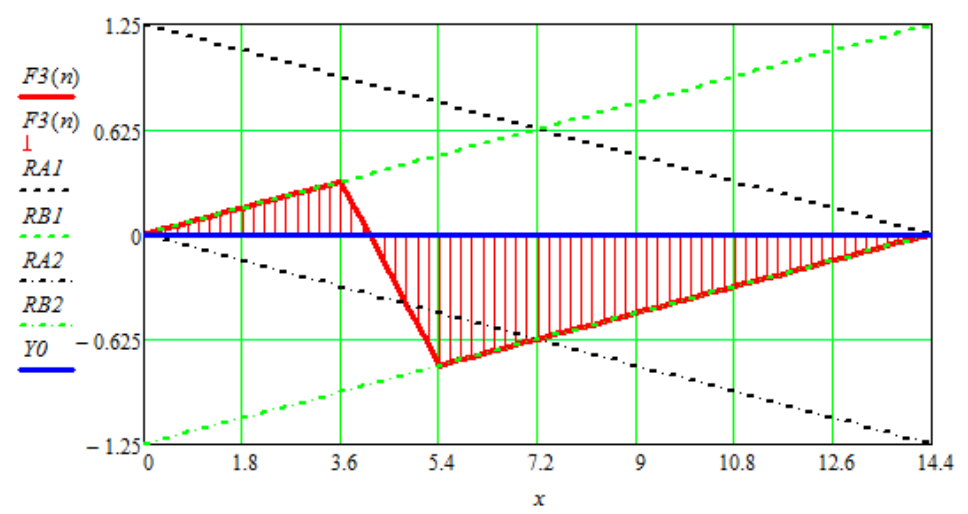

Fig. 4. Influence line F3(n) for the section $n=3$

5. Influence lines F4(n) for diagonal rods of a truss $\mathrm{S} 4(\mathrm{n})$ in any section $\mathrm{n}$ : - in program form (program 4) 


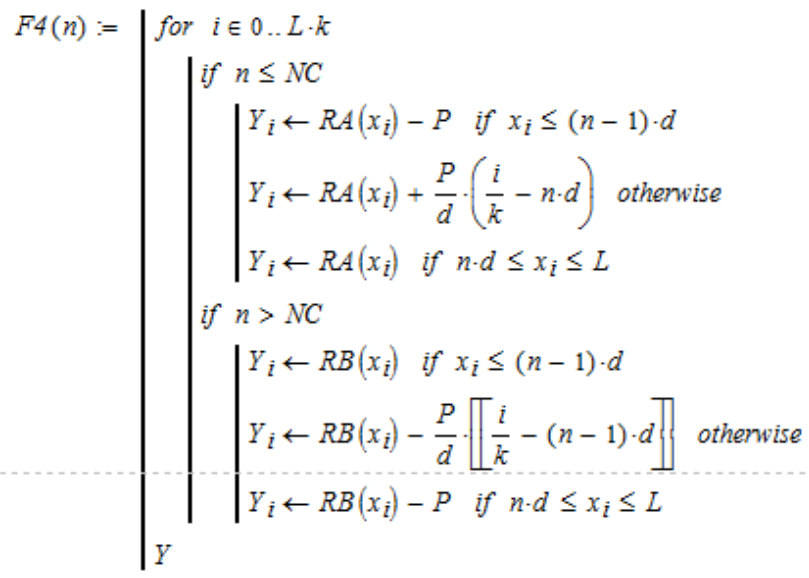

Prog. 4. Program of the influence lines modeling- $F 4(n)$ in the section $\mathrm{n}$ from the position of unit load $x$.

- in a graphical form, for example, in the section (Figure 5)

$$
n:=3
$$

$$
\begin{aligned}
& R B:=P \cdot \frac{x}{L} \quad R A:=P \cdot\left(1-\frac{x}{L}\right) \quad R B 1:=R B-1 \quad R A 1:=R A-1 \\
& \max (F 3(n))=0.625 \quad \min (F 3(n))=-0.25
\end{aligned}
$$

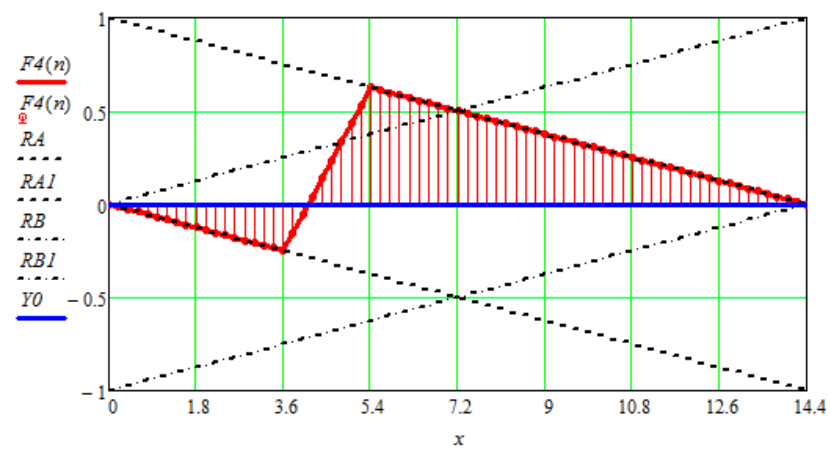

Fig. 5. Influence line $F 4(n)$ for the section $n=3$

6. Influence lines $\mathrm{F} 4 \mathrm{c}(\mathrm{n})$ for diagonal rods of a truss $\mathrm{S} 4(\mathrm{n})$ in any section $\mathrm{n}$ : - in program form (program 5)

$$
F 4 c:=\mid \begin{aligned}
& \text { for } i \in 0 \ldots N \\
& \mid \begin{array}{l}
x_{i} \leftarrow i \cdot d \\
Y_{i} \leftarrow 0 \text { if } 0 \leq x_{i}<(N C-1) \cdot d \\
Y_{i} \leftarrow \frac{x_{i}-(N C-1) \cdot d}{d} \text { if }(N C-1) \cdot d \leq x_{i} \leq N C \cdot d \\
Y_{i} \leftarrow 0 \text { if } x_{i}>(N C+1) \cdot d \\
\left(\begin{array}{l}
Y \\
x
\end{array}\right)
\end{array}
\end{aligned}
$$

Prog.5. Program of the influence lines modeling $-F 2(n)$ in the section $\mathrm{n}$ from the position of unit load - 
- in a graphical form, for example, in the section (Figure 5)

$n:=2$

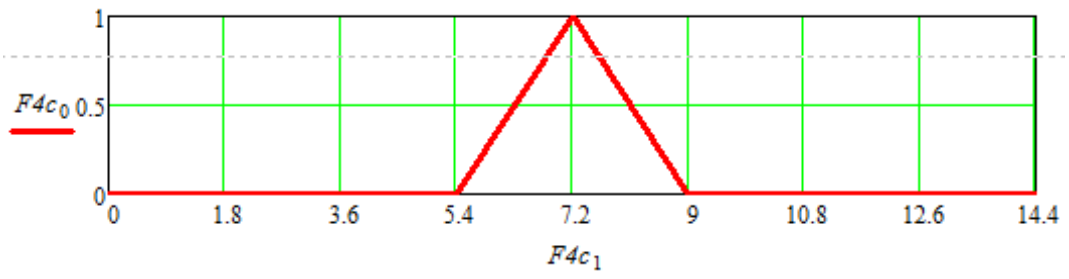

Fig. 6. Influence line F2(n) for the section $n=1$

7. The maximum and minimum values of internal efforts in every rod of a bridge crane truss each section in a tabular form $n:=1 . . N$

\begin{tabular}{|c|c|c|c|c|c|c|}
\hline & $\min (F l(n))$ & $\max (F 2(n))$ & $\max \left(F 3(n)_{1}\right)$ & $\min \left(F 3(n)_{1}\right.$ & $\min (F 4(n))$ & $\max (F 4(n)$ \\
\hline 1 & 0 & 0.656 & -0.122 & -0.122 & 0 & 0.875 \\
\hline 2 & -0.656 & 1.125 & 0.017 & 0.017 & -0.125 & 0.75 \\
\hline 3 & -1.125 & 1.406 & 0.017 & 0.017 & -0.25 & 0.625 \\
\hline 4 & -1.406 & 1.5 & 0.017 & 0.017 & -0.375 & 0.5 \\
\hline 5 & -1.5 & 1.406 & -0.017 & -0.017 & -0.375 & 0.5 \\
\hline 6 & -1.406 & 1.125 & -0.017 & -0.017 & -0.25 & 0.625 \\
\hline 7 & -1.125 & 0.656 & -0.017 & -0.017 & -0.125 & 0.75 \\
\hline 8 & -0.656 & 0 & -0.017 & -0.017 & 0 & 0.875 \\
\hline
\end{tabular}

\section{Conclusion}

The main results of scientific research have been obtained:

- analytical expressions of load beam displacements representation, the maximum sagging stresses in any load beam cross-section depending on the positions of the load cart;

- algorithm and complex of programs of calculation and representation of load beam displacements, the maximum sagging stresses in any load beam cross-section in Mathcad system.

The proposed procedures of modeling of load beam displacements, the maximum sagging stresses in any cross-section of a gantry crane load beam from moving load cart allow to reduce time and cost for the performance of such calculations at least in ten times and effectively to carry out the research when the load beam parameters, load cart parameters and materials change.

\section{References}

1. R. Hibber. Engineering Mechanics: Principles of Statics and Dynamics (Pearson Press, 2006)

2. T. Simankina, I. Kibireva, A. Mottaeva, M. Gusarova, Advances in Intelligent Systems and Computing 983, 138-145 (2019)

3. G.B. Arfken, H.J. Weber, F.E. Harris, M. Gusarova, Mathematical Methods for Physicists International Student Edition: Academic press, (2005) 
4. B. Jerman, J. Kramar, International Journal of Mechanical Sciences 46(5), 729-750 (2004)

5. B. Jerman, J. Kramar, International Journal of Mechanical Sciences 50(3), 490-500 (2008)

6. Ju, F., \& Choo, YS. Dynamics Characteristic of Tower Cranes, (2003)

7. E. Kreyszig, Advanced engineering mathematics (John Wiley\& Sons, 2007)

8. A. Maczynski, S. Wojciech, Nonlinear Dynamics 32(3), 259-290 (2003)

9. R.L. Neitzel, N.S. Seixas, K.K. Ren, Applied Occupational and Environmental Hygiene 16(12), 1106-1117 (2001)

10. G. Sun, M. Kleeberger, J. Liu, Mechanism and machine theory 40(4), 447-466 (2005)

11 M. Torkar, B. Arzenek, Engineering FailureAnalysis 9(2), 227-233 (2002)

12. E.L. Wilson, Three-Dimensional Static and Dynamic Analysis of Structures (Computers and Structures, Inc., Berkeley, CA., 1996)

13. F. Bear. E. Jonston, Vector Mechanics for Engineers I - Static, II - Dynamics, 9th Ed (Tata McGraw Hill, 2011)

14. I. Karnovsky, O. Lebed, Advanced Methods of Structural Analysis. Springer Science + Business Media, (2010) 